Aim of the study: Azacitidine is a hypomethylating agent which is used in the treatment of myelodysplastic syndromes, acute myeloid leukemia and chronic myelomonocytic leukemia. Because of good tolerance to the drug, azacitidine can be administered both during hospitalization and in an outpatient setting.

The aim of our retrospective analysis was to assess the efficacy of azacitidine treatment in patients with a myelodysplastic syndrome and with acute myeloid leukemia who had received treatment in hospital and in an ambulatory care setting. Offsets in the course of azacitidine administration and discontinuations of treatment have a negative impact on patients' response to the therapy.

Material and methods: The study included 31 patients. Sixteen patients received azacitidine in an ambulatory care setting, 15 patients within their hospitalization.

Results: A hematologic response was achieved in $48 \%$ of the patients. Forty-one percent of the cycles were delayed. In an outpatient setting, $62 \%$ of the cycles were administered systematically, while during hospitalization the patients received $54 \%$ of cycles on time. Administrative problems caused the delay of $26 \%$ of the cycles.

Conclusions: Azacitidine has a high tolerance level and a high safety profile which allows for its use in an outpatient care setting. Outpatient administration of azacitidine is feasible and safe without compromising efficacy.

Key words: myelodysplastic syndrome, azacitidine, outpatient treatment.

Contemp Oncol (Pozn) 2015; 19 (6): 467-470 DOI: $10.5114 /$ wo. 2015.56653

\section{Azacitidine in outpatient treatment - single center experience}

\author{
Justyna Rybka ${ }^{1}$, Ewa Stefanko ${ }^{1}$, Aleksandra Bogucka-Fedorczuk ${ }^{1}$ \\ Aleksandra Butrym ${ }^{1,2}$, Rafał Poręba ${ }^{3}$, Kazimierz Kuliczkowski ${ }^{1}$, \\ Tomasz Wróbel
}

${ }^{1}$ Department of Hematology, Blood Neoplasms and Bone Marrow Transplantation, Wroclaw Medical University, Wroclaw, Poland

2Department of Physiology, Wroclaw Medical University, Wroclaw, Poland

${ }^{3}$ Department of Internal Medicine, Wroclaw Medical University, Wroclaw, Poland

\section{Introduction}

Myelodysplastic syndromes (MDS) are a heterogeneous group of bone marrow stem cell diseases characterized by ineffective hematopoiesis leading to peripheral blood cytopenias, complications such as bleeding and an increased risk of leukemic transformation. The incidence of MDS in Europe is $1.8 / 100,000$, while the 5 -year survival rate is approximately $29 \%$ [1].

Azacitidine is a pyrimidine antimetabolite, a hypomethylating agent, which is used in the treatment of patients with MDS, acute myeloid leukemia (AML), a 20-30\% blast cell count in the bone marrow and chronic myelomonocytic leukemia (CMML) with a 10-29\% blast count in the bone marrow [2, 3]. As demonstrated in the AZA-001 trials, azacitidine substantially increases the overall survival (OS) rate in patients with high-risk MDS as compared to conventional therapy [4]. Based on data from randomized clinical trials, it was also found that azacitidine contributes to a reduction of cytopenia and the frequency of the transfusion or blood products in patients with lower-risk MDS $[5,6]$. Since azacitidine's mechanism of action is cycle dependent, it is crucial to follow the schedule of administration.

Unfortunately, in daily clinical practice there are problems with the timely administration of scheduled azacitidine cycles. Azacitidine can be used both in hospitalized patients and in those patients who qualify for treatment outside the hospital ward. The possibility of ambulatory azacitidine administration positively affects the patients' quality of life. Thanks to outpatient treatment, it is easier to maintain the punctuality of consecutive cycles. The survey data that have been collected in 27 hematology-oncology centers in Poland show that approximately $40 \%$ of patients undergoing azacitidine therapy could be treated on an outpatient basis. The survey also found that approximately $24 \%$ of azacitidine packs have been used outside of hospital wards, i.e., within outpatient therapy.

The aim of our retrospective study was to assess the efficacy of treatment with azacitidine in patients with MDS and AML who were undergoing treatment in hospital and in an outpatient setting at the Department of Hematology, Blood Neoplasms and Bone Marrow Transplantation at the Medical University in Wroclaw.

\section{Material and methods}

The retrospective analysis comprised 31 patients (20 men and 11 women) treated with azacitidine in the Department of Hematology, Blood Neoplasms and Bone Marrow Transplantation at the Medical University in Wroclaw in 2008-2015. The median age of the patients was 63 (age range: 41-85). Twenty-seven patients were diagnosed with MDS and 4 with secondary AML (after MDS diagnosis). According to the International Prognostic Scoring System (IPSS), 7 patients were diagnosed with high risk, 16 patients with 
intermediate-2 risk, and 4 patients with intermediate-1 risk. Fifteen patients were treated with azacitidine in the hospital and 16 patients received azacitidine on an outpatient basis. Twenty-three patients required regular substitution of blood products before initiating therapy with azacitidine.

Detailed patient characteristics are shown in Table 1.

\section{Statistical analysis}

Statistical analysis was performed based on the statistical program STATISTICA 12 (StatSoft Poland). The arithmetic mean (X) and standard deviations (SD) of the studied parameters were calculated for quantitative variables. The distribution of variables was tested by the Shapiro-Wilk test. For quantitative variables, the Mann-Whitney test was used for further analysis. The results for qualitative variables were expressed as percentages. For qualitative variables, the maximum likelihood ratio chi-square test was used for further statistical analysis. In order to determine the probability of survival, the Kaplan-Meier curves were analyzed. Statistical significance was defined as $p<0.05$

\section{Results}

\section{Treatment}

In the entire analyzed population of patients, a total of 274 cycles of azacitidine were administered. Azacitidine was administered at the standard dose of $75 \mathrm{mg} / \mathrm{m}^{2} / \mathrm{d}$ for 7 days. The median number of cycles of azacitidine in hospitalized patients was 6 (range: 1-9), while in patients undergoing therapy on an outpatient basis, the median number of cycles was 9 (range: 4-23). In patients treated in chemotherapy ambulatory day-care units, $62 \%$ of the daily cycles of azacitidine were administered as planned. In the group of hospitalized patients, the administration schedule of the cycles was followed in $54 \%$ of the cases. This difference was statistically significant $(p=0.004)$. In

Table 1. Clinical data of patients

\begin{tabular}{|c|c|}
\hline \multicolumn{2}{|l|}{31 patients } \\
\hline Gender & $20 \mathrm{M} / 11 \mathrm{~F}$ \\
\hline Age & 63 (range: 41-85) \\
\hline Diagnosis & $\begin{array}{l}\text { MDS RAEB } 2-20 \\
\text { MDS RAEB } 1-6 \\
\text { MDS RARS }-1 \\
\text { AML }-4\end{array}$ \\
\hline IPSS & $\begin{array}{l}\text { High - } 7 \\
\text { Intermediate }-2-16 \\
\text { Intermediate }-1-4\end{array}$ \\
\hline Pretreatment hemoglobin level & 8.55 g/dl (range: 6.6-14.2) \\
\hline Pretreatment platelet count & $65 \times 10^{9} /$ ( range: $\left.21-223\right)$ \\
\hline $\begin{array}{l}\text { Median blast count in bone } \\
\text { marrow }\end{array}$ & 13 (range: 11-30) \\
\hline Median count of cytopenias & 2 \\
\hline
\end{tabular}

MDS - myelodysplastic syndromes; RAEB - refractory anemia with excess blasts; RARS - refractory anemia with ringed sideroblasts; $A M L$ - acute myeloid leukemia; IPSS - International Prognostic Scoring System the whole group, 112 cycles (41\%) were delayed, including 50 cycles (45\%) for less than 7 days and 62 cycles (55\%) for over 7 days. The reason for the delay of $26 \%$ of the azacitidine treatment cycles was administrative problems (16\% in hospitalized patients and $10 \%$ in an outpatient setting). Fifteen percent of the cycles in hospitalized patients and $20 \%$ of the cycles in patients treated in chemotherapy ambulatory day-care units were postponed due to infections. The $10 \%$ of delay episodes in an outpatient setting and $15 \%$ of delay episodes in hospital were due to hematological toxicity. In the whole group, patients' personal and family problems were the reasons for delayed administration of $8 \%$ of azacitidine cycles.

\section{Response to treatment}

A hematological response (hematological improvement - HI) to treatment occurred in 15 patients (48\%). Five patients (16\%) achieved complete remission (complete remission - CR) and 3 patients (10\%) achieved partial remission (partial remission - PR). The median number of azacitidine cycles after which patients achieved a platelet response was 3 (range: 2-6), the median number of cycles with a granulocytic response was 5 (range: $3-7$ ), while the median number of cycles with a red cell response was 4 (range: 3-6). Transformation to acute leukemia was confirmed in 16 patients. The median number of azacitidine cycles before the diagnosis of $\mathrm{AML}$ was 9 (range: 5-24).

\section{Toxicity}

In 15 patients (48\%) treated with azacitidine, there were side effects of the therapy. These included infections in 6 patients (40\%), febrile neutropenia in 4 patients (27\%), anemia in 3 patients (20\%) and thrombocytopenia in 2 patients (13\%). In 3 patients (1 patient treated on an outpatient basis and in 2 hospitalized patients) the toxic complications necessitated a lowered dose of azacitidine. Five patients (33\%) required additional hospitalization due to an infection or a need for a blood transfusion.

\section{Survival rates}

The median OS for the entire study population was 12.5 months (range: 4-47 months). The median OS for patients treated with azacitidine in an outpatient setting was 16 months (range: 4-47 months), and for patients receiving treatment within the hospital it was 12 months (range: 4-30 months). This difference was statistically significant $(p<0.05)$. The median progression-free survival (PFS) for the study population was 7 months (range: 2-20 months).

The results are shown in Fig. 1.

\section{Discussion}

Azacitidine is currently a very important therapeutic option for patients diagnosed with MDS, AML, and CMML. Its hypomethylating efficiency is dependent on the duration of treatment, and premature discontinuation of treatment affects the response rate and the survival rate of the treated patients [7]. Due to its relatively low toxicity and treat- 
ment possibilities for outpatient care, azacitidine is widely used in older patients with MDS or AML. There are several dosing schedules for administering azacitidine, but as one study showed, efficiency with a satisfactory tolerance is achieved when using azacitidine in 7-day cycles every 28 days [8]. Unfortunately, in everyday clinical practice, it is not always possible to strictly follow administration schedules for subsequent cycles of azacitidine.

The results from the large, multi-center, prospective AVIDA clinical trials, which evaluated patients with MDS treated with azacitidine in a "real life" setting, were published in 2014. The analysis involved 421 patients with a median age of 75. In $81 \%$ of patients, azacitidine was used at a dose of $75 \mathrm{mg} / \mathrm{m}^{2}$. The median of cycles was 5.61 in patients who achieved a hematological response, including $45 \%$ with a red blood cell response, $44 \%$ with a platelet response and $26 \%$ with a granulocyte response. The median patient population observation time was 7.6 months, and the most common reasons for discontinuing the therapy were death (27.5\%), adverse events occurring during treatment (18.8\%) and progression of the disease (4\%). Among the adverse events during the azacitidine therapy, cytopenias, malaise and gastrointestinal disorders occurred [9].

To date, one study which evaluated the cause of the discontinuation or delay of azacitidine therapy has been published. Andrea Tendas et al. analyzed a population of 21 patients with MDS, AML and CMML. The entire group of patients received a total of 199 cycles of azacitidine. 31\% of cycles were not administered in due time, including a delay of under 7 days in $15 \%$ of cycles, under 14 days in $8 \%$ of cycles and over 14 days in $8 \%$ of cycles. The most common causes of the delay in therapy were infections ( $27 \%$ of the cycles), organizational problems in hospital units ( $27 \%$ of the cycles), and hematologic toxicity (20\% of the cycles) [10]. Similar results were obtained in the AZA-001 study, in which $46 \%$ of the azacitidine cycles were delayed [2].

Our retrospective analysis of 31 patients treated with azacitidine aimed to compare the population of patients receiving treatment in an outpatient setting and during hospitalization. In the entire group of patients, $41 \%$ of cycles of azacitidine were delayed. This result is higher than the results presented by Tendas et al., who noted a delay in therapy in $31 \%$ of cycles [10]. In the study group of our ambulatory patients, $62 \%$ of the cycles of azacitidine were administered as planned. In the hospitalized patients, the number of azacitidine cycles that the patients received during the time period was lower and concerned $54 \%$ of the cycles. Twenty-six percent of the cycles were delayed due to administrative problems, mainly related to a lack of beds in the inpatient treatment ward.

In Tendas's publication, the hospital units' organizational problems contributed to an offset in the administration of up to $27 \%$ of the azacitidine cycles. In our study group, $48 \%$ of the patients had toxic complications of the treatment mainly characterized by cytopenias and infections. Thirty-three percent of the patients required additional hospital admissions due to the need for a transfusion or the treatment of an infection. In the trials carried out by Tendas et al., infections and hematological toxicity

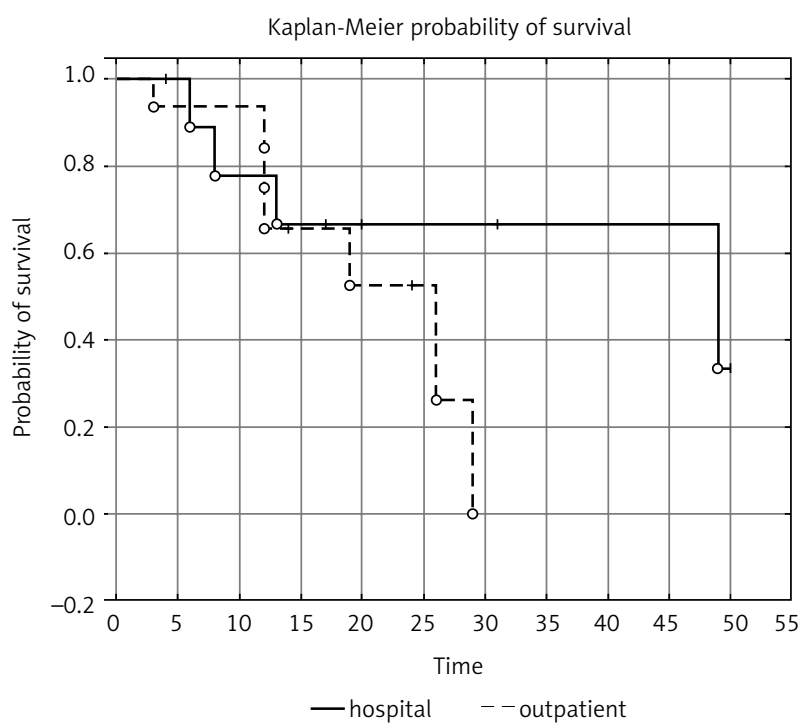

Fig. 1. Survival curves in patients treated with azacitidine

were the cause of delay of $47 \%$ of the cycles [10]. In the patient population analyzed in our study, a hematological response was achieved in $48 \%$ of the patients. This result was lower than in the AVIDA multi-center study, where a hematological response was confirmed in 61\% of the patients, but similar to the AZA-001 study, where HI was $49 \%$ (9.4).

In 27 hematology-oncology centers in Poland, a survey was conducted on the use of azacitidine in an outpatient hospital setting. Respondents reported the possibility of administering azacitidine in about $40 \%$ of patients eligible for treatment outside the hospital (unpublished data). The administration of azacitidine in day-care outpatient units brings many benefits. It makes it easier to maintain the punctuality of scheduled cycles. In order to reduce the risk of complications in the form of infections, one should consider preventive anti-infective treatment. As we have shown in our study, for patients receiving azacitidine within outpatient care, the majority of the cycles were administered as planned. The incidence of complications in the form of cytopenias and infections was comparable in patients treated in ambulatory care units and in hospitalized patients.

The effectiveness of azacitidine largely depends on its administration at appropriate times. Complications after treatment are one of the reasons for the postponement of individual cycles. Still, we should undertake to reduce other causes for the delay of the therapy, especially administrative and locational problems in hospital units.

The authors declare no conflict of interest.

\section{References}

1. Visser O, Trama A, Maynadie M, et al. Incidence, survival and prevalence of myeloid malignancies in Europe. Eur J Cancer 2012; 48 : 3257-66. 
2. Silverman LR, Fenaux P, Mufi GJ, et al. Continued azacitidine therapy beyond time of first response improves quality of response in patients with higher-risk myelodysplastic syndromes. Cancer 2011; 117: 2697-702.

3. Tendas A, Cupelli L, Siniscalchi A, et al. Azacitidine in chronic myelomonocytic leukemia: an effective and manageable approach. Meditter J Hematol Infect Dis 2014; 6: e2014020.

4. Fenaux P, Mufti GJ, Hellstrom-Lindberg E, et al. Efficacy of azacitidine compared with that of conventional care regimens in the treatment of higher risk myelodysplastic syndromes: a randomized, open-label, phase III study. Lancet Oncol 2009; 10: 223-232.

5. Silverman LR, Demakos EP, Peterson BL, et al. Randomized controlled trial of azacitidine in patients with the myelodysplastic syndrome: a study of the cancer and leukemia group B. J Clin Oncol 2002; 20: 2429-40.

6. Lyons RM, Cosgriff TM, Modi SS, et al. Hematologic response to three alternative dosing schedules of azacitidne in patients with myelodysplastic syndromes. J Clin Oncol 2009; 27: 1850-56.

7. Voso MT, Breccia M, Lunghi M, et al. Rapid loss of response after withdrawal of treatment with azacitidine: a case series in patients with higher-risk myelodysplastic syndromes or chronic myelomonocytic leukemia. Eur J Haematol 2013; 90: 345-8.

8. Garcia-Delgado R, de Miguel D, Bailén A, et al. Effectiveness and safety of different azacitidine dosage regimens in patients with myelodysplastic syndromes or acute myeloid leukemia. Leuk Res 2014; 38: 744-50

9. Grinblatt DL, Sekeres MA, Komrokji RS, Swern AS, Sullivan KA, Narang M. Patients with myelodysplastic syndromes treated with azacitidine in clinical practice: the AVIDA registry. Leuk Lymph 2014; 56: 887-95.

10. Tendas A, Lissia MF, Piccioni D, et al. Obstacles to adherence to azacitidine administration schedule in outpatient myelodysplastic syndrome and related disorders. Support Care Cancer 2015; 23: 303-5.

\section{Address for correspondence}

Justyna Rybka MD, PhD

Department of Hematology, Blood Neoplasms

and Bone Marrow Transplantation

Wroclaw Medical University

Pasteura 4

50-367 Wroclaw, Poland

tel. +48717842576

fax +48713270963

e-mail: rybka.justyna@o2.pl

Submitted: 25.09 .2015

Accepted: 18.12 .2015 